\title{
Decreased insulin resistance in diabetic patients by influencing Sirtuin 1 and Fetuin-A following supplementation with ellagic acid: a randomized controlled trial
}

\author{
Mahnaz Ghadimi ${ }^{1}$, Farshad Foroughi ${ }^{2}$, Sima Hashemipour ${ }^{3}$, Mohammadreza Rashidi Nooshabadi ${ }^{4}$, \\ Mohammad Hossein Ahmadi ${ }^{5}$, Mojtaba Ghadimi Yari ${ }^{6}$, Maria Kavianpour ${ }^{7^{*+}}$ \\ and Hossein Khadem Haghighian ${ }^{1,3^{*}+}$
}

\begin{abstract}
Background: The beneficial effects of polyphenols have been reported. This study aimed to investigate the effect of oral Ellagic acid (EA) supplement on insulin resistance (IR) and Fetuin-A and serum sirtuin1 (SIRT1) in type 2 diabetics.

Methods: In this double-blind, randomized clinical trial, 44 diabetic patients were selected. Patients were assigned to the intervention group ( 22 subjects) and placebo (22 subjects) and received a capsule containing $180 \mathrm{mg}$ of EA per day or placebo for eight weeks, respectively. At the beginning and end of the study, anthropometric indices, fasting plasma glucose (FPG), plasma insulin level, IR, Fetuin-A, and SIRT1 were measured. Statistical analysis was performed using SPSS software.
\end{abstract}

Results: At the beginning and end of the study, there was no significant difference between the two groups regarding anthropometric indices $(P>0.05)$. At the end of the survey, EA supplementation significantly reduced FPG, insulin, $I R$, and Fetuin-A and increased SIRT1 levels compared with the placebo group $(P<0.05)$. However, these changes were not significant in the placebo group $(P>0.05)$.

Conclusion: EA with antioxidant properties plays an essential role in reducing the macrovascular and microvascular complications of diabetes by reducing inflammation and insulin resistance.

Trial registration The protocol of this clinical trial is registered with the Iranian Registry of Clinical Trials (http://www. IRCT.IR, identifier: IRCT20141025019669N13)

Keywords: Ellagic acid, Glycemic index, Insulin resistance, Diabetes type 2

*Correspondence: Kavianpour.maria@gmail.com; khademnut@yahoo.com ${ }^{\dagger}$ Maria Kavianpour and Hossein Khadem Haghighian contributed equally to this work

${ }^{1}$ Department of Nutrition, School of Health, Qazvin University of Medical

Sciences, Qazvin, Iran

${ }^{7}$ Department of Tissue Engineering and Applied Cell Sciences, Faculty of Advanced Technologies in Medicine, Tehran University of Medical Sciences, Tehran, Iran

Full list of author information is available at the end of the article

\section{Background}

Diabetes mellitus is one of the five leading causes of death in the world, characterized by impaired insulin secretion, insulin activity, or both, and is divided into two categories: type 1 diabetes and type 2 diabetes [1]. Results of studies show that in 2030, 552 million people will have type 2 diabetes. Insulin function in diabetics and its subsequent resistance or sensitivity to insulin are the cases that, despite significant research and success in this field,

c) The Author(s) 2021. This article is licensed under a Creative Commons Attribution 4.0 International License, which permits use, sharing, adaptation, distribution and reproduction in any medium or format, as long as you give appropriate credit to the original author(s) and the source, provide a link to the Creative Commons licence, and indicate if changes were made. The images or other third party material in this article are included in the article's Creative Commons licence, unless indicated otherwise in a credit line to the material. If material is not included in the article's Creative Commons licence and your intended use is not permitted by statutory regulation or exceeds the permitted use, you will need to obtain permission directly from the copyright holder. To view a copy of this licence, visit http://creativeco mmons.org/licenses/by/4.0/. The Creative Commons Public Domain Dedication waiver (http://creativecommons.org/publicdomain/ zero/1.0/) applies to the data made available in this article, unless otherwise stated in a credit line to the data. 
many aspects of it, especially in post-receptor defects, have not been fully identified for the medical research [2]. According to scientific reports, there are factors and cytokines that play a stimulating or inhibitory role in insulin function. Recently, Sirtuin1 and Fetuin-A have been considered by researchers in diabetic patients [3].

Silent information regulator 1 (SIRT1) proteins, known as sirtuins, were identified as genetic silencing factors. Mammalian sirtuins proteins are homogeneous from Sir2 of yeast and prolong the lifespan in yeast [4]. Mammals have seven types of sirtuins from SirT1 to SirT7. SIRT1 is a NAD-dependent histone deacetylase that senses oxidative stress conditions and increases a protective cellular response. To regulate transcription, it transfers acetyl groups from the amino acids $\mathrm{N}$-acetyl lysine in DNA histones. It is also expressed in the brain, heart, liver, pancreas, skeletal muscle, spleen, and adipose tissue. SIRT1 plays a vital role in the pathology, progression, and treatment of several diseases, including neurological disorders such as Alzheimer's disease, cardiovascular disease, metabolic disease, and aging-related disease [5].

SIRT1 inhibits oxidative stress through deacetylation of p53, NF- $\mathrm{kB}$, and Forkhead box class $\mathrm{O}$ family member proteins (FoxOs) and induction of manganese Superoxide dismutase (SOD). Deacetylation of p53 and FOXO by SIRT1 reduces transcription activity and subsequently reduces stress-induced apoptosis [6]. Scientific investigations have reported that SIRT1 negatively regulates transcription factors such as NF- $\mathrm{kB}$ in the nucleus by lysine destabilization on histones and other non-histone proteins. Also, SIRT1 protects cells from oxidative stress by increasing catalase activity. Studies have shown that SIRT1 activity in retinal tissue in diabetic rats is reduced [7]. In cells with insulin resistance, SIRT1 is significantly reduced, resulting in impaired glucose tolerance [8].

Fetuin-A ( $\alpha 2$-Heremans-Schmid glycoprotein) $\mathrm{A}$ is a 64-kilo daltonic glycoprotein with multiple functions that are produced in the liver and then secreted in the serum. Fetuin-A is a suitable inhibitor of Pathological vascular calcification; however, it is an important factor in insulin resistance [9]. Fetuin-A is located in the $3 q 27$ gene in the human genome, which is a prone site for diabetes and metabolic syndrome. Numerous studies have shown that Fetuin-A levels in patients with T2D and obesity increased compared to the healthy control group. Reports have also shown that Fetuin-A levels are associated with an increased risk of metabolic syndrome. Fetuin-A inhibits the insulin receptor Tyrosine kinase in skeletal muscle and liver cells and stops insulin signals transmission, thereby causing insulin resistance in these target tissues [10]. Fetuin-A in mice with insulin resistance caused by a high-fat diet led to a low regulation of TLR4-mediated inflammatory signaling in adipose tissue
[11]. According to the above contents, increasing SIRT1 levels and decreasing Fetuin-A levels is a new therapeutic approach to treating metabolic disorders such as type 2 diabetes. Polyphenols stimulate the expression of SIRT1, which protects cells from oxidative stress [12]. The reason why polyphenols increase the level of SIRT1 in the body is not well known, but it can be related to their antioxidant effect [13].

Ellagic acid (EA) is a polyphenol found in various fruits, including berries, raspberries, pomegranates, and walnuts [14]. Due to this polyphenol's antioxidant property and the possibility of its favorable effect on SIRT1 and Fetuin-A levels and reduced insulin resistance, this study aimed to investigate the effect of EA oral supplement on SIRT1, Fetuin-A, and insulin resistance in patients with type 2 diabetes.

\section{Materials and methods}

\section{Ethics}

The current research project's approval was done as a randomized, double-blind, placebo-controlled clinical trial by the ethical committee of Qazvin University of Medical Sciences (IR.QUMS.REC.1398.079) and with final registration and approval on the site of clinical trials in Iran (IRCT20141025019669N13). The study was performed on people with type 2 diabetes at the Endocrinology and Metabolism Clinic of Qazvin University of Medical Sciences. According to the Human Studies Protocol, the participants in the research project signed a consent form.

\section{Inclusion and exclusion criteria}

People with type 2 diabetes (hemoglobin A1c (HbA1c) $\geq 6 \%$; or fasting plasma glucose (FPG) $\geq 126 \mathrm{mg} /$ dl (7 mmol/l); or 2-hp glucose $\geq 200 \mathrm{mg} / \mathrm{dl}$ (11.1 mmol/l), satisfaction with participation in clinical trials, no change in diet, physical activity and medication were the inclusion criteria. Having a body mass index in the range of obesity, pregnancy and lactation, insulin injection, diseases that interfere with the treatment of diabetes, use of antioxidant supplements, and dissatisfaction with participation in clinical research intervention are the criteria for not including the study. Also, changes in lifestyle (diet and physical activity) and treatment methods, and any reported side effects of the supplement were exclusion criteria.

\section{Patients}

At the beginning of this intervention study, with the scientific opinion of the expert and clinical consultant of the present investigation, 54 people with type 2 diabetes of both sexes, and aged 24-55, a total of 54 people were introduced to the researcher to explain the research 
process fully and finally enter the study. After complete explanations and additional introduction, 44 people entered the study with complete satisfaction. After complete explanations and introduction of the dietary supplement used in the project, 44 people entered the study with complete satisfaction.

\section{Study design}

To investigate the effect of $180 \mathrm{mg}$ Ellagic acid supplementation daily for 60 days on insulin resistance and serum SIRT1 and Fetuin levels, this randomized, double-blind, placebo-controlled clinical trial study was designed, approved, and performed. Patients who entered the research project were fully informed and justified about the study and its implementation steps, then the required information such as demographic characteristics, medical history, and current drugs as well as anthropometric data [height, weight, and body mass index (BMI)] was collected by the project researcher using a questionnaire and appropriate tools. Grouping of patients in the intervention (EA) $(n=22)$ and placebo $(n=22)$ groups was done randomly (by randomized block allocation) and after equalizing their entry in the group in terms of age, sex and weight. Each person received an ellagic acid capsule $(180 \mathrm{mg})$ or a placebo capsule containing wheat flour once daily with meals. The color, shape, and size of the supplement capsule were similar to that of the placebo capsule. In this study, the patient, researcher, and specialist physician were blind to supplement and placebo. Capsules prepared by someone else outside the study in A and B groups were placed in the same package so that the investigator would be unaware of the capsules' contents. The effective selective dose for Ellagic acid supplement was taken from an article by Mario Falsaperela et al. The supplement was purchased from a SupplementSpot, and the placebo was made by School of Pharmacy, Tabriz University of Medical Sciences. To control for confounding effects of diet and physical activity, at the beginning and at the end of the study, patients were interviewed by a 3-day dietary recall questionnaire, and subjects with moderate physical activity level were enrolled. Three-day food recalls were used to assess dietary intake, and the Nutritionist IV program (San Bruno, CA) modified for Iranian food composition was used for estimating the dietary intake of participants. Also, to evaluate the physical activity, we used the International Physical Activity Questionnaire (IPAQ) [15]. Data from the IPAQ were converted to metabolic equivalent-minutes/week using existing guidelines [16]. Patients were followed up to control their ellagic acid capsules' consumption and prevented from falling out once every seven days by telephone. At the end of the study, each person should return the bottle containing their supplement for count capsules. Patients who consumed less than $10 \%$ of capsules were removed from the study.

\section{Biochemical measurements}

At the beginning and end of the study, ten ccs of venous blood samples were taken, and after the separation of serum, SIRT and Fetuin levels were measured in the plasma of the participants. Blood samples were collected into tubes with and without EDTA. Blood samples without EDTA Centrifuged (Beckman Avanti J-25, USA) at a rate of $3000 \mathrm{rpm}$ for $10 \mathrm{~min}$ in order to the separation of serum. Blood samples were kept at $-70{ }^{\circ} \mathrm{C}$ (Snijdes, Germany) in Diabetes Research Center and then transferred to the laboratory of Velayat Hospital of Qazvin University of Medical Sciences, and the measurements were carried out. Fasting plasma glucose (FPG) concentration was measured by the enzymatic method using an Abbot ModelAclyon 300, USA auto analyzer with Pars-Azmone kit (Tehran, Iran). Plasma insulin was measured by using a chemiluminescent immunoassay method (LIAISON analyzer (310360) Diasorin S.P.A., Verecelli, Italy). Insulin resistance (HOMA-IR) was calculated according to the following formula: HOMA-IR = (fasting insulin (U/ $\mathrm{ml}) \times$ FPG $(\mathrm{mg} / \mathrm{dl}) / 405)$ [17]. Serum levels of SIRT1 and Fetuin-A in participants' plasma were measured using a special kit and by ELISA method (Diameter, Italy and Bioassay Technology Laboratory, China).

\section{Sample size}

The level of the Malondialdehyde (MDA) factor was used to calculate sample size before and after the administration of pomegranate extract in the study of Hosseini et al. [18] using the following formula:

$$
\mathrm{N}=\left[\left(\mathrm{Z}_{1-\alpha / 2}+\mathrm{Z}_{1-\beta}\right)^{2}\left(\mathrm{SD}_{1}^{2}+\mathrm{SD}_{2}^{2}\right)\right] / \Delta^{2}
$$

where a (type 1 error) is 0.05 , b (type 2 error) is 0.2 , S1 and $\mathrm{S} 2$ are the variances of MDA and $\Delta$ represent the different means of MDA. Thus, the power for detecting differences between the two groups for various outcomes in the present study was $80 \%$. The sample size was obtained, 13 in each group. Considering 30\% probable drop-out, the sample size was considered 22 . In this research, 44 diabetic patients were studied.

\section{Statistical analyses}

Statistical analyses were conducted using SPSS version 20. Results were presented as mean \pm SD, and the normality of data distribution was assessed by the Kolmogorov-Smirnov test. To comparison of mean biochemical variables before and after intervention in each group was used paired sample t-test statistical method 
and to compare the variables between two groups was used independent sample t-test method. Differences were considered statistically significant at $\mathrm{P}<0.05$.

\section{Result}

In this study, 44 people with type 2 diabetes participated, which were divided into two groups of 22 people (intervention and placebo). In this investigation, one patient from each group was excluded from the study for personal reasons. Patient compliance in this study was $95.45 \%$. The duration of this study was eight weeks. No side effects were reported in the study. Patients' individual characteristics are shown in Table 1. There is no statistically significant difference between the two groups in terms of individual characteristics. The mean ages of participants in the intervention and placebo groups were $47 \pm 5.06$ and $44.04 \pm 6.88$ years old, respectively $(\mathrm{P}>0.05)$. Also, there was no significant difference between the two groups in terms of anthropometric factors at the beginning of the study. The mean and standard deviation of weight (67.23 \pm 10.24 vs. $68.95 \pm 9.02)$, Height (162.43 \pm 10.59 vs. $163.71 \pm 9.12)$, and BMI $(25.32 \pm 1.07$ vs. $25.61 \pm 0.86)$ were in the intervention and placebo groups, respectively. Also, there was no significant difference in the amount of physical activity. The amount of food intake is given in Table 2. There were no statistically significant differences at the beginning and

Table 1 The comparison of baseline characteristics of the participants

\begin{tabular}{llll}
\hline Variable & $\begin{array}{l}\text { Mean } \pm \text { SD } \\
\text { Placebo }(\mathbf{n}=\mathbf{2 1})\end{array}$ & $\begin{array}{l}\text { Mean } \mathbf{S D} \\
\text { Ellagic acid }(\mathbf{n}=\mathbf{2 1})\end{array}$ & $\mathbf{P 1}$ \\
\hline Age (years) & $44.04 \pm 6.88$ & $47 \pm 5.06$ & 0.701 \\
Height $(\mathrm{cm})$ & $163.71 \pm 9.12$ & $162.43 \pm 10.59$ & 0.814 \\
Weight $(\mathrm{kg})$ & & & \\
Before & $68.95 \pm 9.02$ & $67.23 \pm 10.24$ & 0.621 \\
After & $68.49 \pm 9.21$ & $66.98 \pm 10.31$ & 0.59 \\
P2 & 0.78 & 0.619 & \\
Body mass index $\left(\mathrm{Kg} / \mathrm{m}^{2}\right)$ & & \\
Before & $25.61 \pm 0.86$ & $25.32 \pm 1.07$ & 0.562 \\
After & $25.43 \pm 0.9$ & $25.22 \pm 1.09$ & 0.604 \\
P2 & 0.719 & 0.807 & \\
Physical activity & & & 0.132 \\
Before & $37.27 \pm 2.78$ & $36.15 \pm 2.99$ & 0.108 \\
After & $37.5 \pm 3.31$ & $36.43 \pm 2.89$ & \\
P2 & 0.429 & 0.49 & \\
Metformin dose & $1643.17 \pm 377.88$ & $1610.4 \pm 362.07$ & \\
\hline
\end{tabular}

Data are expressed as means \pm SD

P1: Comparison of the mean of baseline characteristics between the two groups of Ellagic acid and placebo (Independent samples t-test)

P2: Comparison of mean of baseline characteristics in each group at baseline and end of study (Paired samples t-test)
Table 2 The comparison of the dietary intake at the baseline and the end of the study in participants

\begin{tabular}{|c|c|c|c|}
\hline Variables & $\begin{array}{l}\text { Mean } \pm \text { SD } \\
\text { Placebo }(n=21)\end{array}$ & $\begin{array}{l}\text { Mean } \pm S D \\
\text { Ellagic acid }(n=21)\end{array}$ & P1 \\
\hline \multicolumn{4}{|c|}{ Energy (kcal) } \\
\hline Baseline & $2057.71 \pm 230.546$ & $2034.41 \pm 286.84$ & 0.405 \\
\hline End & $2043.49 \pm 241.269$ & $2026.38 \pm 292.2$ & 0.3 \\
\hline P2 & 0.58 & 0.48 & \\
\hline \multicolumn{4}{|l|}{ Protein (g) } \\
\hline Baseline & $82.19 \pm 16.3$ & $80.81 \pm 23.11$ & 0.55 \\
\hline End & $81.07 \pm 21.03$ & $78.27 \pm 18.01$ & 0.41 \\
\hline P2 & 0.68 & 0.6 & \\
\hline \multicolumn{4}{|c|}{ Carbohydrate (g) } \\
\hline Baseline & $263.61 \pm 62.19$ & $260.71 \pm 49.13$ & 0.59 \\
\hline End & $261.39 \pm 51.09$ & $259.14 \pm 52.2$ & 0.52 \\
\hline P2 & 0.63 & 0.69 & \\
\hline \multicolumn{4}{|l|}{ Fat (g) } \\
\hline Baseline & $77.5 \pm 16.22$ & $76.3 \pm 15.07$ & 0.801 \\
\hline End & $75.18 \pm 24.18$ & $74.17 \pm 15.07$ & 0.705 \\
\hline P2 & 0.309 & 0.41 & \\
\hline \multicolumn{4}{|c|}{ Saturated fatty acids (g) } \\
\hline Baseline & $24.08 \pm 6.31$ & $23.27 \pm 6.17$ & 0.77 \\
\hline End & $23.1 \pm 6.07$ & $22.06 \pm 5.17$ & 0.662 \\
\hline P2 & 0.47 & 0.6 & \\
\hline \multicolumn{4}{|c|}{ Monounsaturated fatty acid (g) } \\
\hline Baseline & $28.09 \pm 8.14$ & $26.45 \pm 7.3$ & 0.807 \\
\hline End & $28.22 \pm 7.12$ & $26.65 \pm 5.3$ & 0.59 \\
\hline P2 & 0.63 & 0.74 & \\
\hline \multicolumn{4}{|c|}{ Polyunsaturated fatty acid (g) } \\
\hline Baseline & $25.28 \pm 7.64$ & $25.56 \pm 7.03$ & 0.82 \\
\hline End & $24.15 \pm 5.18$ & $24.27 \pm 5.17$ & 0.86 \\
\hline P2 & 0.354 & 0.29 & \\
\hline \multicolumn{4}{|l|}{ Fiber (g) } \\
\hline Baseline & $9.27 \pm 1.03$ & $8.95 \pm 1$ & 0.48 \\
\hline End & $8.65 \pm 1.44$ & $8.07 \pm 1.2$ & 0.401 \\
\hline P2 & 0.28 & 0.25 & \\
\hline \multicolumn{4}{|c|}{ Vitamin C (mg) } \\
\hline Baseline & $69.14 \pm 20.19$ & $68.47 \pm 13.27$ & 0.74 \\
\hline End & $67.47 \pm 23.01$ & $68.03 \pm 14.37$ & 0.52 \\
\hline P2 & 0.583 & 0.641 & \\
\hline \multicolumn{4}{|c|}{ Vitamin E (IU) } \\
\hline Baseline & $8.24 \pm 1.01$ & $7.66 \pm 1.24$ & 0.456 \\
\hline End & $7.53 \pm 1.21$ & $7.87 \pm 1.01$ & 0.11 \\
\hline P2 & 0.353 & 0.84 & \\
\hline \multicolumn{4}{|l|}{ Selenium } \\
\hline Baseline & $121.77 \pm 41.02$ & $120 \pm 25.07$ & 0.481 \\
\hline End & $120.16 \pm 44.53$ & $119.44 \pm 19.61$ & 0.3 \\
\hline P2 & 0.347 & 0.491 & \\
\hline
\end{tabular}

Data are expressed as means \pm SD

P1: Comparison of the mean of dietary intake between the two groups of Ellagic acid and placebo (Independent samples t-test)

P2: Comparison of mean of baseline characteristics in each group at baseline and end of study (Paired samples t-test) 
end of the study in terms of energy intake, macronutrients, and micronutrients. The effects of Ellagic acid supplementation on FPG, Insulin, IR, STRT1, and, Fetuin-A are shown in Table 3 . There were no statistically significant differences between intervention and placebo at the beginning and end of the study. At the end of the study, EA supplementation reduced FPG, Insulin, IR, and Fetuin-A levels and increased SIRT1 levels $(\mathrm{P}<0.05)$. In the placebo group, the differences in mean levels of SIRT1, Fetuin-A, and IR at the end of the study were not significant.

\section{Discussion}

This study was designed to investigate the effect of EA on insulin resistance, SIRT1, and Fetuin-A in patients with type 2 diabetes. This study showed that the administration of $180 \mathrm{mg}$ of ellagic acid for eight weeks significantly

Table 3 Changes in baseline to endpoint measures for SIRT1, Fetuin-A and HOMA-IR in two groups

\begin{tabular}{|c|c|c|c|}
\hline Variables & $\begin{array}{l}\text { Mean } \pm \text { SD } \\
\text { Placebo }(n=21)\end{array}$ & $\begin{array}{l}\text { Mean } \pm \text { SD } \\
\text { Intervention }(n=21)\end{array}$ & P1 \\
\hline \multicolumn{4}{|l|}{ FBS (mg/dl) } \\
\hline Baseline & $163.28 \pm 7.90$ & $160.80 \pm 8.94$ & 0.811 \\
\hline End & $162.52 \pm 8.54$ & $123.47 \pm 9.73$ & 0.022 \\
\hline P2 & 0.91 & 0.011 & \\
\hline Mean changes & $-0.76 \pm 0.64$ & $-37.33 \pm 0.79$ & 0.032 \\
\hline \multicolumn{4}{|l|}{ Insulin ( $\mu \mathrm{U} / \mathrm{ml})$} \\
\hline Baseline & $16.08 \pm 0.77$ & $15.83 \pm .88$ & 0.53 \\
\hline End & $16.00 \pm .84$ & $12.16 \pm .96$ & .031 \\
\hline P2 & 0.77 & 0.03 & \\
\hline Mean changes & $-0.08 \pm 0.07$ & $-3.67 \pm 0.08$ & 0.039 \\
\hline \multicolumn{4}{|l|}{ HOMA-IR } \\
\hline Baseline & $6.49 \pm .63$ & $6.30 \pm .72$ & 0.45 \\
\hline End & $6.43 \pm .68$ & $3.72 \pm .58$ & 0.038 \\
\hline $\mathrm{P} 2$ & 0.59 & 0.02 & \\
\hline Mean changes & $-0.06 \pm 0.05$ & $-2.58 \pm 0.14$ & 0.042 \\
\hline \multicolumn{4}{|l|}{ Fetuin-A ( $\mu \mathrm{g} / \mathrm{ml})$} \\
\hline Baseline & $123.58 \pm 24.11$ & $119.8 \pm 25.07$ & 0.21 \\
\hline End & $121.63 \pm 24.01$ & $101.31 \pm 19.07$ & 0.031 \\
\hline P2 & 0.159 & 0.022 & \\
\hline Mean changes & $-1.95 \pm 0.1$ & $-18.49 \pm 6$ & 0.033 \\
\hline \multicolumn{4}{|l|}{ SIRT1 (ng/mL) } \\
\hline Baseline & $6.04 \pm 0.04$ & $5.96 \pm 0.05$ & 0.46 \\
\hline End & $6.02 \pm 0.03$ & $6.94 \pm 0.09$ & 0.033 \\
\hline P2 & 0.66 & 0.01 & \\
\hline Mean changes & $-0.02 \pm 0.01$ & $+0.98 \pm 0.04$ & 0.046 \\
\hline
\end{tabular}

Data are expressed as means \pm SD

P1: Comparison of the mean of dietary intake between the two groups of Ellagic acid and placebo (Independent samples t-test)

P2: Comparison of mean of baseline characteristics in each group at baseline and end of study (Paired samples t-test) reduced blood sugar, insulin, and insulin resistance. Babaian et al. Observed that daily intake of $240 \mathrm{ml}$ of unsweetened pomegranate juice reduced insulin resistance in patients with type 2 diabetes. In contrast, no significant changes were observed in blood sugar and fat levels. Low doses of EA in pomegranate juice or short study time are the reasons for the lack of significant effect on glycemic indexes [19]. Also, Kanchana et al. studied the anti-diabetic effect of EA in diabetic mice caused by streptozotocin for 35 days. The results of their study showed that rats with diabetes had a significant increase in plasma glucose, $\mathrm{HbA}_{1} \mathrm{c}$, and hexokinase levels and decreased insulin and peptide $C$, glycogen (liver and muscle), and glucose 6 phosphatase activity in the liver and kidneys compared with healthy mice. Oral administration of EA for 35 days significantly reduced blood glucose levels, HbA1c, the enzyme hexokinase, glycogen (liver and muscle), and the activity of glucose 6 phosphatases in the liver and the kidneys [20]. Based on the reported results of the scientific studies, it has been suggested that a possible mechanism for lowering blood sugar may be by enhancing insulin secretion from pancreatic beta cells or by transferring more blood glucose to the surrounding tissue [21]. In diabetic animals, EA may increase insulin secretion or glucose uptake [20]. Insulin resistance as a lack of sensitivity to insulin function in skeletal muscle, liver, and adipose tissue. After consuming carbohydrates, insulin stimulates glycogenesis in muscles and the liver and inhibits lipolysis in adipose tissue [22]. When IR occurs, the altered insulin response results in the secretion of free fatty acids from adipose tissue and the storage of triglycerides in the muscles and liver. Oxidative stress and activation of inflammatory pathways and lipolysis caused by macrophages promote insulin resistance and non-alcoholic fatty liver disease (NAFLD). Advances in IR can lead to dyslipidemia, high blood pressure, excessive blockage of blood vessels, and fibrinolysis disorders. IR increases the level of oxidized-LDL and cardiovascular disease incidence, particularly in patients with type 2 diabetes [23].

Also, administering $180 \mathrm{mg}$ of EA for eight weeks significantly increased SIRT1 and reduced Fetuin-A in patients with type 2 diabetes. Reducing excess body fat causes a significant increase in the levels of this enzyme [24]. Studies have shown that lowering SIRT1 in mice impairs glucose tolerance. Mice fed a high-fat diet have lower levels of SIRT1 in the pancreas and liver and may be associated with insulin resistance. Inhibition of SIRT1 disrupts insulin signaling by interfering with the phosphorylation of the insulin receptor and glycogen synthase [25]. Khalili et al. assessed the Lactobacillus casei effects on Glycemic response, Serum Sirtuin1, and Fetuin-A Levels in Patients with 
Type 2 Diabetes. They reported that the levels of SIRT1 and Fetuin-A were significantly affected by probiotic consumption. In their investigation, the $\mathrm{L}$. casei supplementation significantly increased SIRT1 and decreased Fetuin-A levels at the end of the trial [3]. SIRT plays an essential role in many biological processes, including metabolism, cell proliferation, tumor suppression, genomic stability, and aging prevention. One way to reduce oxidative stress in the body and create balance is to increase SIRT secretion [26]. By increasing the expression of antioxidant and anti-inflammatory enzymes and reducing pro-inflammatory factors such as NF-KB, this enzyme ultimately benefits the body by increasing its antioxidant capacity and reducing the destructive effects of ROS [4]. SIRT1 deacetylates lysine 310 of RelA/p65 subunit of NF-kB, an important subunit for for activation of transcription of proinflammatory genes, triggers inflammatory processes.This NF-kB signaling pathway is the prototypical one involved in inflammation $[27,28]$. In this way, it can be said that by affecting the secretion of this enzyme, it can help treat diabetic patients by reducing insulin resistance $[7,29]$. Janda et al. showed that serum Fetuin-A levels were associated with blood sugar deposition in the arteries and that Fetuin-A had been shown to affect the AGE/RAGE pathway in the presence of inflammatory molecules [30]. Excessive Fetuin-A expression increases levels and activates $\mathrm{NF}-\mathrm{KB}$ and other inflammatory cytokines. Fetuin-A levels are also positively correlated with CRP levels. This protein is activated by free fatty acids (FFAs) and triggers pre-inflammatory reactions [31]. In addition, in order to promoting insulin resistance in type 2 diabetic patients, Fetuin-A can also prevent the change of RAGE ligand with (HMGB1), which is responsible the release and absorption of several cytokines, adhesive molecules, and cheomokines. Activation of the RAGE signal cascade pathway has been shown to balance HMGB1 expression in tumor necrosis factor (TNFa) and interleukin 1 (IL-1) [32].

Having parallel groups to eliminate the effect of the drug and controlling confounding factors such as physical activity and diet are the strengths of the study. Also, according to the research of the researchers of the present study, supplementation with pure EA was performed for the first time on the IR index. However, it should be noted that in order to determine the clinical effects and cost-effectiveness of this supplement in type 2 diabetic patients, it is necessary to have more participants and study several doses, because in this plan due to existing limitations such as financial, the number of participants and the unit dose studied, the results of this study are statistically interpreted.

\section{Conclusion}

In conclusion, the results of this study indicated that eight weeks of supplementation with EA, $180 \mathrm{mg} /$ day, reduced the levels of blood sugar, Insulin, IR, and FetuinA and increased SIRT1 in patients with type 2 diabetes. These results provide evidence to support the view that polyphenol antioxidant group with reducing the complications of diabetes can play an important role in helping to control the condition of diabetes, including reducing the dose of medications used. Nevertheless, further studies are needed to provide additional evidence.

\section{Abbreviations \\ BMI: Body Mass Index; DM: Diabetes mellitus; EA: Ellagic acid; FPG: Fasting blood sugar; FoxOs: Forkhead box class O family member proteins; $\mathrm{HbA} 1 \mathrm{c}$ : Hemoglobin A1c; IPAQ: International Physical Activity Questionnaire; IR: Insulin resistance; MDA: Malondialdehyde; NAFLD: Non-alcoholic fatty liver disease; ROS: Reactive oxygen species; SIRT1: Sirtuin 1; SIRT1: Silent information regula- tor 1; SOD: Superoxide dismutase.}

\section{Acknowledgements}

The authors would like to thank the all of the participants who completed the study protocol.

\section{Authors' contributions}

HKH guarantor of the article; MG, FF, MK performed the analysis of the data and statistics, and wrote the manuscript; MG, SH, MRN, MHA and MGY designed the study and interpreted the results; FF, MK and HKH supervised the statistical analyses; MG, SH and MRN collected the data; HKH obtained funding for the study. All authors revised the manuscript critically. All authors have approved the final version of the article, including the authorship list. All authors read and approved the final manuscript.

\section{Funding}

This work was financially supported by a Grant (Number: IR.QUMS.

REC.1398.079) from Vice-Chancellor for Research Affairs of Qazvin University of Medical Sciences, Qazvin, Iran. The role of the funding body was in the design of the study, data collection, analysis, and interpretation of data.

\section{Availability of data and materials}

The datasets used and/or analyzed during the current study are available from the corresponding author upon reasonable request.

\section{Ethics approval and consent to participate}

We confirm that any aspect of the work covered in this manuscript that has involved either human patient has been conducted with the ethical approval of all relevant bodies, in Ethical committee of Qazvin University of Medical Science, Qazvin, Iran by grant number of IR.QUMS.REC.1398.079. On the other hand, the protocol was registered in IRCT by the number IRCT20141025019669N13. All the eligible and volunteered subjects had been written consent for supplementary care before research.

\section{Consent for publication}

Not applicable.

\section{Competing interests}

The authors declare that they have no competing interests.

\section{Author details}

${ }^{1}$ Department of Nutrition, School of Health, Qazvin University of Medical Sciences, Qazvin, Iran. ${ }^{2}$ Department of Immunology, School of Medicine, Qazvin University of Medical Sciences, Qazvin, Iran. ${ }^{3}$ Metabolic Diseases Research Center, Research Institute for Prevention of Non-Communicable Diseases, Qazvin University of Medical Sciences, Qazvin, Iran. ${ }^{4}$ Department of Pharmacology, School of Pharmacy, Ahvaz Jundishapur University of Medical Sciences, Ahvaz, Iran. ${ }^{5}$ Department of Laboratory Sciences, School of Allied Medical 
Sciences, Qazvin University of Medical Sciences, Qazvin, Iran. ${ }^{6}$ Determinants of Chemistry, Amirkabir University of Technology, Tehran, Iran. ${ }^{7}$ Department of Tissue Engineering and Applied Cell Sciences, Faculty of Advanced Technologies in Medicine, Tehran University of Medical Sciences, Tehran, Iran.

Received: 2 November 2020 Accepted: 22 January 2021 Published online: 05 February 2021

\section{References}

1. Collier A, Meney C, Hair M, Cameron L, Boyle JG. Cancer has overtaken cardiovascular disease as the commonest cause of death in Scottish type 2 diabetes patients: a population-based study (The Ayrshire Diabetes Follow-up Cohort study). J Diabetes Investig. 2020;11 (1):55-61.

2. Goto A, Takao T, Yoshida Y, Kawazu S, Iwamoto Y, Terauchi Y. Causes of death and estimated life expectancy among people with diabetes: a retrospective cohort study in a diabetes clinic. J Diabetes Investig. 2020;11(1):52-4.

3. Khalili L, Alipour B, Jafar-Abadi MA, Faraji I, Hassanalilou T, Abbasi MM, et al. The effects of lactobacillus casei on glycemic response, serum sirtuin 1 and fetuin-a levels in patients with type 2 diabetes mellitus: a randomized controlled trial. Iran Biomed J. 2019;23(1):68.

4. Broussy S, Laaroussi H, Vidal M. Biochemical mechanism and biological effects of the inhibition of silent information regulator 1 (SIRT1) by EX-527 (SEN0014196 or selisistat). J Enzyme Inhib Med Chem. 2020;35(1):1124-36.

5. Chong Z, Matsuo $\mathrm{H}$, Onoue $\mathrm{S}$, Yamamoto $\mathrm{H}$, Ito $\mathrm{H}$, Katakura $\mathrm{Y}$. Identification of polyphenols that repair the ultraviolet-B-induced DNA damage via SIRT1-dependent XPC/XPA activation. J Funct Foods. 2019;54:119-27.

6. Mohammed ET, Hashem KS, Abdelazem AZ, Foda FA. Prospective protective effect of ellagic acid as a SIRT1 activator in iron oxide nanoparticleinduced renal damage in rats. Biol Trace Element Res. 2020;198:177-88.

7. Duarte DA, Mariana Ap BR, Papadimitriou A, Silva KC, Amancio VHO, Mendonça JN, et al. Polyphenol-enriched cocoa protects the diabetic retina from glial reaction through the sirtuin pathway. J Nutr Biochem. 2015;26(1):64-74.

8. Wang R, Xie Y, Qiu J, Chen J. The effects of dexmedetomidine in a rat model of sepsis-induced lung injury are mediated through the adenosine monophosphate-activated protein kinase (AMPK)/silent information regulator 1 (SIRT1) pathway. Med Sci Monit Int Med J ExpClin Res. 2020;26:e919213-21.

9. Fatima F, Ahsan N, Nasim A, Alam F. Association of fetuin-A with dyslipidemia and insulin resistance in type-II Diabetics of Pakistani population. Pak J Med Sci. 2020;36(2):64.

10. Zhang L-Y, Qu X-N, Sun Z-Y, Zhang Y. Effect of liraglutide therapy on serum fetuin A in patients with type 2 diabetes and non-alcoholic fatty liver disease. Clin Res HepatolGastroenterol. 2020;44:674-80.

11. Dholey Y, Chaudhuri A, Sen CS. An integrated in silico approach to understand protein-protein interactions: human meprin- $\beta$ with fetuin-A. J BiomolStruct Dyn. 2020;38(7):2080-92.

12. Domazetovic V, Marcucci G, Falsetti I, Bilia AR, Vincenzini MT, Brandi ML, et al. Blueberry juice antioxidants protect osteogenic activity against oxidative stress and improve long-term activation of the mineralization process in human osteoblast-like SaOS-2 cells: involvement of SIRT1. Antioxidants. 2020;9(2):125.

13. Sarubbo F, Esteban S, Miralles A, Moranta D. Effects of resveratrol and other polyphenols on Sirt1: relevance to brain function during aging. CurrNeuropharmacol. 2018;16(2):126-36.

14. Kang I, Buckner T, Shay NF, Gu L, Chung S. Improvements in metabolic health with consumption of ellagic acid and subsequent conversion into urolithins: evidence and mechanisms. AdvNutr. 2016;7(5):961-72.

15. Tran VD, Do VV, Pham NM, Nguyen CT, Xuong NT, Jancey J, et al. Validity of the international physical activity questionnaire-short form for application in Asian countries: a study in Vietnam. Eval Health Prof. 2020;43(2):105-9.

16. Pathare N, Conroy J, Gillard A, Hansen M. Physical activity: levels, knowledge, and attitudes of physical therapy students in the United States. CardiopulmPhysTher J. 2020;31(2):57-65.

17. Najdi RA, Hagras MM, Kamel FO, Magadmi RM. A randomized controlled clinical trial evaluating the effect of Trigonella foenum-graecum (fenugreek) versus glibenclamide in patients with diabetes. Afr Health Sci. 2019;19(1):1594-601.

18. Hosseini B, Saedisomeolia A, Wood LG, Yaseri M, Tavasoli S. Effects of pomegranate extract supplementation on inflammation in overweight and obese individuals: a randomized controlled clinical trial. Complement TherClinPract. 2016;22:44-50.

19. Babaeian S, Ebrahimi-Mameghani M, Niafar M, Sanaii S. The effect of unsweetened pomegranate juice on insulin resistance, high sensitivity c-reactive protein and obesity among type 2 diabetes patients. J Ardabil Univ Med Sci. 2013;13(1):7-15.

20. Malini P, Kanchana G, Rajadurai M. Antibiabetic efficacy of ellagic acid in streptozotocin-induced diabetes mellitus in albino wistar rats. Asian J Pharm Clin Res. 2011;4(3):124-8.

21. Keller MP, Rabaglia ME, Schueler KL, Stapleton DS, Gatti DM, Vincent M, et al. Gene loci associated with insulin secretion in islets from nondiabetic mice. J Clin Invest. 2019;129(10):4419-32.

22. Qaid MM, Abdelrahman MM. Role of insulin and other related hormones in energy metabolism — a review. Cogent Food Agric. 2016;2(1):1267691.

23. Yuan T, Yang T, Chen H, Fu D, Hu Y, Wang J, et al. New insights into oxidative stress and inflammation during diabetes mellitus-accelerated atherosclerosis. Redox Biol. 2019;20:247-60.

24. Milne JC, Lambert PD, Schenk S, Carney DP, Smith JJ, Gagne DJ, et al. Small molecule activators of SIRT1 as therapeutics for the treatment of type 2 diabetes. Nature. 2007:450(7170):712-6.

25. Sun C, Zhang F, Ge X, Yan T, Chen X, Shi X, et al. SIRT1 improves insulin sensitivity under insulin-resistant conditions by repressing PTP1B. Cell Metab. 2007;6(4):307-19.

26. D'Onofrio N, Servillo L, Balestrieri ML. SIRT1 and SIRT6 signaling pathways in cardiovascular disease protection. Antioxid Redox Signal. 2018;28(8):711-32.

27. Salminen A, Kauppinen A, Suuronen T, Kaarniranta K. SIRT1 longevity factor suppresses NF-KB-driven immune responses: regulation of aging via NF-KB acetylation? BioEssays. 2008;30(10):939-42.

28. Saha SK, Pietras EM, He JQ, Kang JR, Liu SY, Oganesyan G, et al. Regulation of antiviral responses by a direct and specific interaction between TRAF3 and Cardif. EMBO J. 2006;25(14):3257-63.

29. Pacifici F, Di Cola D, Pastore D, Abete P, Guadagni F, Donadel G, et al. Proposed tandem effect of physical activity and sirtuin 1 and 3 activation in regulating glucose homeostasis. Int J Mol Sci. 2019;20(19):4748.

30. Janda K, Krzanowski M, Gajda M, Dumnicka P, Jasek E, Fedak D, et al. Vascular effects of advanced glycation end-products: content of immunohistochemically detected AGEs in radial artery samples as a predictor for arterial calcification and cardiovascular risk in asymptomatic patients with chronic kidney disease. Dis Mark. 2015. https://doi. org/10.1155/2015/153978.

31. Hameed I, Masoodi SR, Mir SA, Nabi M, Ghazanfar K, Ganai BA. Type 2 diabetes mellitus: from a metabolic disorder to an inflammatory condition. World J Diabetes. 2015;6(4):598.

32. Wang $X$, Sun X, Niu M, Zhang X, Wang J, Zhou C, et al. RAGE silencing ameliorates neuroinflammation by inhibition of p38-NF-KB signaling pathway in mouse model of Parkinson's disease. Front Neurosci. 2020;14:353.

\section{Publisher's Note}

Springer Nature remains neutral with regard to jurisdictional claims in published maps and institutional affiliations. 